\title{
Thermometry of ultracold atoms via nonequilibrium work distributions
}

\author{
T. H. Johnson, ${ }^{1,2,3,{ }^{*}}$ F. Cosco, ${ }^{2,4,5}$ M. T. Mitchison, ${ }^{2,6}$ D. Jaksch, ${ }^{1,2,3}$ and S. R. Clark ${ }^{2,3,7,8}$ \\ ${ }^{1}$ Centre for Quantum Technologies, National University of Singapore, 3 Science Drive 2, 117543 Singapore \\ ${ }^{2}$ Clarendon Laboratory, University of Oxford, Parks Road, Oxford OX1 3PU, United Kingdom \\ ${ }^{3}$ Keble College, University of Oxford, Parks Road, Oxford OX1 3PG, United Kingdom \\ ${ }^{4}$ Turku Centre for Quantum Physics, Department of Physics and Astronomy, University of Turku, FI-20014 Turun yliopisto, Finland \\ ${ }^{5}$ Dip. Fisica, Università della Calabria, 87036 Arcavacata di Rende (CS), Italy \\ ${ }^{6}$ Quantum Optics and Laser Science Group, Blackett Laboratory, Imperial College London, London SW7 2BW, United Kingdom \\ ${ }^{7}$ Department of Physics, University of Bath, Claverton Down, Bath BA2 7AY, United Kingdom \\ ${ }^{8}$ Max Planck Institute for the Structure and Dynamics of Matter, Luruper Chaussee 149, 22761 Hamburg, Germany
}

(Received 21 September 2015; published 23 May 2016)

\begin{abstract}
Estimating the temperature of a cold quantum system is difficult. Usually one measures a well-understood thermal state and uses that prior knowledge to infer its temperature. In contrast, we introduce a method of thermometry that assumes minimal knowledge of the state of a system and is potentially nondestructive. Our method uses a universal temperature dependence of the quench dynamics of an initially thermal system coupled to a qubit probe that follows from the Tasaki-Crooks theorem for nonequilibrium work distributions. We provide examples for a cold-atom system, in which our thermometry protocol may retain accuracy and precision at subnano-Kelvin temperatures.
\end{abstract}

DOI: 10.1103/PhysRevA.93.053619

\section{INTRODUCTION}

Many technological applications utilizing quantum systems, e.g., analog quantum simulators [1], require precise and accurate measurements of their temperature, making thermometry of quantum systems a fundamental task. Conventional thermometry proceeds by bringing a small probe of known temperature dependence into equilibrium with a thermal system and then measuring that probe. For accurate thermometry, this requires that the characteristic energy of the probe is precisely known and tuned near the thermal energy [2]. This can be challenging at the low temperatures relevant for experiments on ultracold atoms.

Instead, the temperature of ultracold gases is often inferred by directly measuring observables whose temperature dependence is well understood. For instance, time-of-flight imaging of the momentum distribution is used to obtain the temperature of weakly interacting cold atoms [3,4]. Meanwhile, for strongly interacting atoms in a lattice, temperature is inferred from fluctuations of lattice-site occupation numbers [5]. However, such an approach is only feasible if the system Hamiltonian or its thermal states are well characterized and sufficiently simple, so that the temperature dependence of observables can be calculated and compared with measurements. Unfortunately, these requirements are frequently unmet in cold-atom experiments, where the system can be strongly correlated and established perturbative or numerical techniques typically fail. What is missing is a generic approach for thermometry of cold atoms that does not need prior understanding of the thermal state.

To fill this gap we return to the idea of bringing probes into contact with an initially thermal quantum system, this time focusing on the ensuing nonequilibrium dynamics. This increasingly studied [6-9] and potentially nondestructive

\footnotetext{
*tomihjohnson@gmail.com
}

approach to investigating quantum systems has been used to analyze the parameters [10] and spectrum [11] of a Hamiltonian, and the non-Markovianity of an open quantum system [12]. Applications of this approach to the thermometry of cold atoms [13-16] show that focusing on nonequilibrium dynamics can avoid the requirement of precisely tuning the characteristic energy of the probe near the thermal energy. However, the particular approaches put forward so far rely on the system having a well-understood Hamiltonian.

In this article we show how to use a nonequilibrium probe to infer the temperature of a cold-atom system, which may in principle have an arbitrary Hamiltonian. Our approach exploits the Tasaki-Crooks theorem [17-20]: a universal temperature-dependent relationship between nonequilibrium work distributions that may be embedded in the state of a qubit probe [21-23]. We demonstrate that this versatile method is naturally suited to thermometry of cold atomic gases, and is both accurate and robust in the presence of imperfect data. Importantly, no detailed knowledge of the internal dynamics of the system is needed. The only requirement is control over the coupling between the system and the two states of the qubit thermometer, which is readily achieved by using an atomic impurity as the probe. Our protocol thus realizes near-ideal thermometry within its domain of applicability, which corresponds to temperatures commensurate with or lower than the characteristic energy scales of the system. This is precisely the temperature regime of greatest interest for cold-atom physics, and also a challenging one for conventional thermometry.

We gauge the accuracy and precision of our protocol by simulating its application to a paradigmatic ultracold-atom system. Specifically, we consider a Bose-Hubbard model (BHM) and localized impurity qubit, as could be realized by cold bosons in an optical lattice and, for example, two internal states of a separately trapped atom of a different species [24-27]. Our protocol maintains accuracy and precision to a few percent in all regimes investigated. This includes when 
the thermal energy is one or two orders of magnitudes lower than the hopping energy of the BHM, where the latter might typically correspond to tens of nano-Kelvin. Moreover, it includes intermediate interaction strengths for which the nature of the thermal state is poorly understood and neither time-of-flight nor number-fluctuation measurements reveal the temperature. This work thus opens the door for thermometry of generic cold-atom systems at extreme temperatures and the technologies, e.g., quantum simulation, that require such thermometry. We begin with a general description of our scheme, before proceeding to a detailed study of its application to the BHM.

\section{DESCRIPTION OF THE PROTOCOL}

\section{A. Nonequilibrium work distributions}

Our thermometry protocol is based on a relationship between distributions of the work done by quenching a system away from equilibrium. We write $P_{Q}(W)$ for the distribution of the work $W$ done on a system, e.g., a cold atomic gas, due to a quench $Q$. In the quench, the parameter $\lambda$ appearing in a system's Hamiltonian $\hat{H}(\lambda)=\hat{H}_{S}+\lambda \hat{V}$ is varied as $\lambda_{Q}(t)$ for $t \in[0, \tau]$ driving the system away from an initial thermal state $\hat{\rho}_{\beta}\left(\lambda_{Q}(0)\right)$. Here $\beta$ is the inverse temperature, $\hat{\rho}_{\beta}(\lambda)=e^{-\beta \hat{H}(\lambda)} / \mathcal{Z}_{\beta}(\lambda)$ is a thermal state of the system, and $\mathcal{Z}_{\beta}(\lambda)=\operatorname{tr}\{\exp [-\beta \hat{H}(\lambda)]\}$ is the corresponding partition function.

The forward distribution $P_{F}(W)$ for some quench $\lambda_{F}(t)$ from $\lambda_{i}$ to $\lambda_{f}$ is related to the backward distribution $P_{B}(W)$ of its reverse $\lambda_{B}(t)=\lambda_{F}(\tau-t)$ from $\lambda_{f}$ to $\lambda_{i}$ via the TasakiCrooks relation [17-20]

$$
\ln \{R(W)\}=\ln \left\{\frac{P_{F}(W)}{P_{B}(-W)}\right\}=\beta(W-\Delta F) .
$$

The ratio $R(W)$ of the work distributions therefore depends only on $\beta$ and one other constant, the free energy difference $\Delta F=F\left(\lambda_{f}\right)-F\left(\lambda_{i}\right)$, with $F(\lambda)=\beta^{-1} \ln \left[\mathcal{Z}_{\beta}(\lambda)\right]$. Note that this relation holds generally for a coherent quench; it is not based on assumptions of linear response or adiabaticity.

\section{B. Qubit interferometry}

To directly measure quantum work distributions [28-30], and thus their ratio $R(W)$, requires overcoming significant challenges, namely the realization of often prohibitively large numbers of difficult projective energy measurements $[31,32]$. We instead consider an indirect approach to measuring $R(W)$ using qubit interferometry [21-23]. The system of interest is brought into contact with a probe qubit, giving total Hamiltonian $\hat{H}_{T}(t)=-(\Delta / 2) \hat{\sigma}_{z}+\hat{H}_{S}+\hat{H}_{I}(t)$. Here $\Delta$ is the difference in energy between the ground and excited states $|\downarrow\rangle$ and $|\uparrow\rangle$ of the qubit, $\hat{H}_{S}$ is the Hamiltonian of the system of interest, and the interaction $\hat{H}_{I}(t)$ takes the form

$$
\hat{H}_{I}(t)=\left[g_{\downarrow}(t)|\downarrow\rangle\left\langle\downarrow\left|+g_{\uparrow}(t)\right| \uparrow\right\rangle\langle\uparrow|\right] \otimes \hat{V} .
$$

The combined system is initialized at time $t=0$ in the state $\hat{\rho}=|s\rangle\langle s| \otimes \hat{\rho}_{\beta}\left(\lambda_{Q}(0)\right)$, with the qubit in some superposition $|s\rangle=s_{\downarrow}|\downarrow\rangle+s_{\uparrow}|\uparrow\rangle$. This could be achieved by first reaching equilibrium with $\beta \Delta \gg 1$ and $g_{\downarrow}(0)=g_{\uparrow}(0)=\lambda_{Q}(0)$, then applying a rotation $\hat{\sigma}_{s}=s_{\uparrow} \hat{\sigma}_{x}+s_{\downarrow} \hat{\sigma}_{z}$ to the qubit. The statedependent couplings $g_{\downarrow}(t)$ and $g_{\uparrow}(t)$ are then both varied according to the quench $\lambda_{Q}(t)$ to be investigated, but with the latter delayed by a time $u$, i.e.,

$$
\begin{aligned}
& g_{\downarrow}(t)= \begin{cases}\lambda_{Q}(t), & 0 \leqslant t \leqslant \tau, \\
\lambda_{Q}(\tau), & \tau \leqslant t \leqslant \tau+u,\end{cases} \\
& g_{\uparrow}(t)= \begin{cases}\lambda_{Q}(0), & 0 \leqslant t \leqslant u, \\
\lambda_{Q}(t-u), & u \leqslant t \leqslant \tau+u .\end{cases}
\end{aligned}
$$

At time $\tau+u$, when both quenches are complete, the qubit has the reduced density operator, in units where $\hbar=1$,

$$
\begin{aligned}
\hat{\rho}_{q}= & \left|s_{\downarrow}\right|^{2}|\downarrow\rangle\left\langle\downarrow\left|+s_{\uparrow}^{*} s_{\downarrow} e^{i \Delta(\tau+u)} \chi_{Q}^{*}(u)\right| \downarrow\right\rangle\langle\uparrow| \\
& +s_{\downarrow}^{*} s_{\uparrow} e^{-i \Delta(\tau+u)} \chi_{Q}(u)|\uparrow\rangle\left\langle\left.\downarrow|+| s_{\uparrow}\right|^{2} \mid \uparrow\right\rangle\langle\uparrow| .
\end{aligned}
$$

Here we have introduced the dephasing function

$$
\chi_{Q}(u)=\operatorname{tr}\left\{\hat{U}_{Q}^{\dagger} e^{i u \hat{H}\left(\lambda_{Q}(\tau)\right)} \hat{U}_{Q} e^{-i u \hat{H}\left(\lambda_{Q}(0)\right)} \hat{\rho}_{\beta}\left(\lambda_{Q}(0)\right)\right\},
$$

where $\hat{U}_{Q}=\mathcal{T} \exp \left[-i \int_{0}^{\tau} d t \hat{H}\left(\lambda_{Q}(t)\right)\right]$ evolves the system according to the time-dependent quench Hamiltonian $\hat{H}\left(\lambda_{Q}(t)\right)$ and $\mathcal{T}$ is the time-ordering operator.

Close examination reveals that $\chi_{Q}(u)$ is none other than the characteristic function, or Fourier transform, of the work distribution [21,22]

$$
P_{Q}(W)=(2 \pi)^{-1} \int d u e^{-i W u} \chi_{Q}(u) .
$$

Hence it is possible to measure $P_{Q}(W)$ from $\chi_{Q}(u)$, and the latter from expected values

$$
\left\langle\hat{\sigma}_{x}\right\rangle+i\left\langle\hat{\sigma}_{y}\right\rangle=\operatorname{tr}_{q}\left\{\left(\hat{\sigma}_{x}+i \hat{\sigma}_{y}\right) \hat{\rho}_{q}\right\}=2 s_{\downarrow}^{*} s_{\uparrow} e^{-i \Delta(\tau+u)} \chi_{Q}(u),
$$

for the qubit state [Eq. (3)] at the end of the interference protocol. In what follows, we set $s_{\downarrow}^{*} s_{\uparrow}=1 / 2$ and $\Delta=0$, with the more general case treated in the Supplemental Material [33].

\section{Thermometry}

The main result of this article is that we are able to use the above relations to systematically, precisely, and accurately infer temperature from realistically noisy data, without any knowledge regarding the thermal states $\hat{\rho}_{\beta}(\lambda)$ of the quantum system. One needs only to have good control over a single qubit and its interaction with the system, which can be achieved by using an atomic impurity as the probe.

Let us analyze these claims in order. Our protocol is robust in the presence of two fundamental sources of error, analyzed in detail in the Supplemental Material [33]. First, it is only possible to estimate $\chi_{Q}(u)$ for a finite number of times $u_{j}$. Here we assume $N_{\text {steps }}$ times $u_{j}=j T / N_{\text {steps }}$ for $j=1, \ldots, N_{\text {steps }}$. Second, each estimate of $\left\langle\hat{\sigma}_{x}\right\rangle$ and $\left\langle\hat{\sigma}_{y}\right\rangle$ used to estimate $\chi_{Q}\left(u_{j}\right)$ will have some error. Here we assume that errors are due to the finite number $N_{\text {meas }}$ of measurements used to estimate each expectation value. However, other known qubit measurement errors can be treated in the same framework.

The first point means that rather than estimating $P_{Q}(W)$, we instead estimate $p_{Q}(W)$, a copy that is subject to spectral 
leakage, due to the finite time-window $T$, and aliasing, due to the discreteness of $u_{j}$. We show that provided typically modest values of $T$ and $N_{\text {steps }}$ are chosen such that $\pi \beta / T$ is on the order of unity and $T / N_{\text {steps }} \ll \tau_{Q \text { deph }}=1 / \sigma_{Q}$, then any effect on the ratio is negligible, i.e., $R(W) \approx p_{F}(W) / p_{B}(-W)$ (see Supplemental Material [33]). Here $\tau_{Q \text { deph }}$ is the delay $u$ needed for the qubit to significantly dephase and is the inverse of $\sigma_{Q}$, the width of the work distribution $P_{Q}(W)$.

The second point means our estimate of $\chi_{Q}\left(u_{j}\right)$ will be an unbiased Gaussian random variable with variance $\left[2-\left|\chi_{Q}\left(u_{j}\right)\right|^{2}\right] / N_{\text {meas }}$, which then propagates linearly into an unbiased Gaussian estimate of $p_{Q}(W)$ with variance scaling as $T^{2} / N_{\text {steps }} N_{\text {meas. }}$. In a Bayesian approach detailed in the Supplemental Material [33], we show how this knowledge, together with the Tasaki-Crooks relation and the non-negativity of the work distributions, can be used to build the probability distribution $\mathcal{P}(\beta)$ for $\beta$ given a set of estimates of $\chi_{Q}\left(u_{j}\right)$. It is also possible to include any prior knowledge of $\beta$ and $\Delta F$, though here we assume no prior knowledge. Our approach is found to be well calibrated and accurate, to a few percent, given a modest number of times $N_{\text {steps }}$ and measurements $N_{\text {meas }}$.

The universality of the Tasaki-Crooks temperature dependence allows the thermometry protocol to be applied in complete ignorance of the quantum system's thermal state $\hat{\rho}_{\beta}(\lambda)$ and even its Hamiltonian $\hat{H}_{S}$. It only needs to be ensured that both states of the impurity couple to the same operator $\hat{V}$ [Eq. (2)], that the coupling strengths $g_{\downarrow}(t)$ and $g_{\uparrow}(t)$ trace the same path with some delay, and that the backwards path mirrors the forward path. Such properties may be understood theoretically in advance or confirmed experimentally by observing how the qubit, in eigenstate $|\downarrow\rangle$ or $|\uparrow\rangle$, behaves when interacting with the system. The choices of perturbing Hamiltonian $\hat{V}$ and quench $\lambda_{Q}(t)$ used for thermometry are arbitrary in principle, since they affect $\Delta F$ not $\beta$. In fact, provided the relationships above hold, the actual values of $\hat{V}$ and $\lambda_{Q}(t)$ need not be known.

In practice, any thermometer benefits from optimization, and our protocol is no exception. In particular, the quench should be chosen so that the fluctuations of the nonequilibrium work are on the order of the temperature or larger. This ensures that the ratio of work distributions, $R(W)$ in Eq. (1), can be accurately and precisely inferred over a large enough range of values of the work $W$ to extract a good straight-line fit for $\beta$. Faced with an unknown system, the experimenter may therefore need to make some adjustments in order to find an appropriate quench. We emphasise that this optimization can be based purely on observations of the qubit evolution, without resorting to direct measurements on the system of interest. Nevertheless, it is clearly important to use a probe qubit whose interaction with the system is highly controllable and well understood over a range of energies.

In the context of cold atomic gases, a probe qubit comprising an atomic impurity satisfies these requirements well. In the following, we consider density-density coupling, as appropriate for an impurity interacting with a host gas of a different species. At low temperatures, this interaction is characterized by a small number of parameters, such as $s$-wave scattering lengths, which may be accurately measured via independent experiments and controlled by means of external fields. Furthermore, the range of interaction energies between the impurity atom and the atomic gas naturally coincides with the characteristic energies of interaction between the gas atoms themselves. It is therefore apparent that the nonequilibrium work fluctuations induced by an atomic impurity are well suited to characterize temperatures that are on the same order or smaller than the natural energy scales of the cold-atom system.

\section{EXAMPLE WITH COLD ATOMS IN A LATTICE}

For concreteness, from here on we take our system to be a cold atomic gas confined by an optical lattice, and the qubit to be formed by two internal states of an impurity atom of a different species, which a strong trap localizes to density $n_{q}(\mathbf{r})$ [24-27]. Both impurity states $|\downarrow\rangle$ and $|\uparrow\rangle$ couple via the contact interaction to the weighted density operator $\hat{V}=\int d \mathbf{r} n_{q}(\mathbf{r}) \hat{\Psi}^{\dagger}(\mathbf{r}) \hat{\Psi}(\mathbf{r})$ with different interaction strengths $g_{\downarrow}$ and $g_{\uparrow}$. Here $\hat{\Psi}^{\dagger}(\mathbf{r})$ and $\hat{\Psi}(\mathbf{r})$ are the field operators for the atoms comprising the system. Thus it is possible to realize a combined Hamiltonian $\hat{H}_{T}$ of the form required for our protocol. The qubit gate $\hat{\sigma}_{s}$, and the measurement of $\hat{\sigma}_{x}$ and $\hat{\sigma}_{y}$, can be performed, e.g., using Rabi pulses combined with state-dependent fluorescing.

The separate time dependence of the coupling strengths $g_{\downarrow}(t)$ and $g_{\uparrow}(t)$ could be controlled by Feschbach resonances [24-27]. Another option is to realize the time dependence of the coupling strengths effectively by changing the properties of their trap, which may be state selective. A further possibility is to forgo using internal states to form the qubit, instead splitting the wave function of an impurity, passing two copies through the system, and then interfering them [34].

For our specific example, we consider a one-dimensional Bose gas in a simple periodic lattice, which reduces to the Bose-Hubbard model [35]

$$
\hat{H}_{S}=-J \sum_{\left\langle j j^{\prime}\right\rangle} \hat{a}_{j}^{\dagger} \hat{a}_{j^{\prime}}+\sum_{j=1}^{M}\left(\frac{U}{2} \hat{a}_{j}^{\dagger} \hat{a}_{j}^{\dagger} \hat{a}_{j} \hat{a}_{j}-\mu \hat{a}_{j}^{\dagger} \hat{a}_{j}\right) .
$$

Here $\hat{a}_{j}^{\dagger}$ and $\hat{a}_{j}$ create and annihilate a particle at site $j$ of a total of $M$, the hopping and interaction energies and chemical potential are written $J, U$, and $\mu$, respectively, and $\left\langle j j^{\prime}\right\rangle$ represents a sum over nearest neighbors. Assuming the impurity to be localized at a single central site $c$, the system's interaction Hamiltonian is $\hat{V}=\eta \hat{a}_{c}^{\dagger} \hat{a}_{c}$, where $\eta=$ $\int d \mathbf{r} n_{q}(\mathbf{r})\left|w_{c}(\mathbf{r})\right|^{2}$ and $w_{c}(\mathbf{r})$ is the Wannier function at the central site.

\section{A. Superfluid phase}

In the superfluid regime $n U / J \ll 1$, with $n$ the number of bosons per site, we can describe the system approximately in terms of phononic Bogoliubov excitations above the uniform condensate of density $n$. To second order in these excitations and terms that create them, the system and interaction Hamiltonians simplify [36], up to a constant, to

$$
\begin{aligned}
\hat{H}_{S} & =\sum_{k} \omega_{k} \hat{b}_{k}^{\dagger} \hat{b}_{k}, \\
\hat{V} & =\eta n+\sum_{k}\left(\eta_{k}^{*} \hat{b}_{k}^{\dagger}+\eta_{k} \hat{b}_{k}\right) .
\end{aligned}
$$



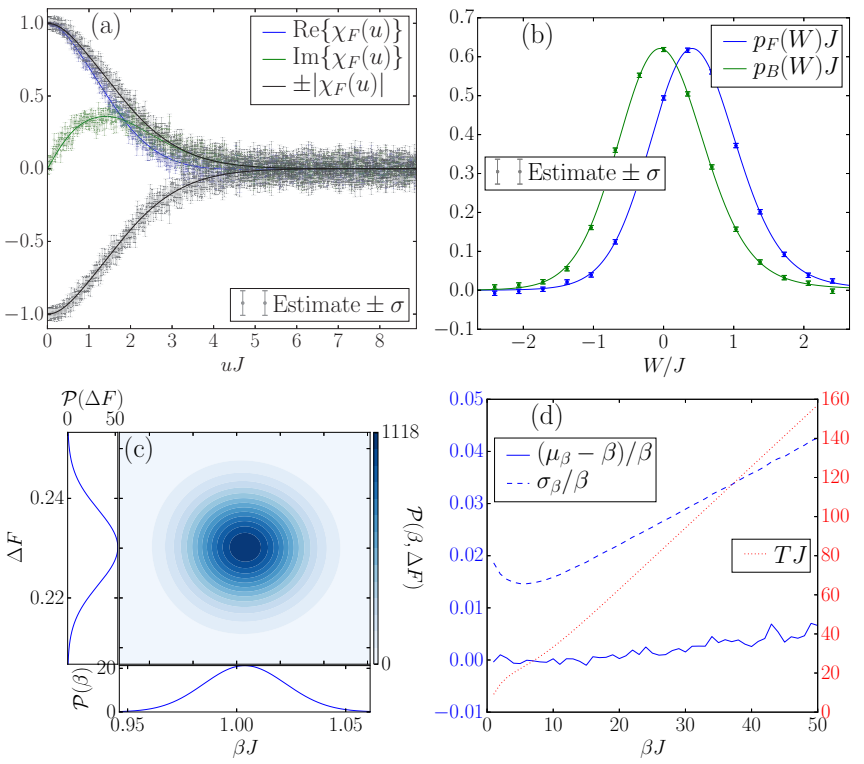

FIG. 1. Superfluid phase. (a) Characteristic function $\chi_{F}(u)$ of the forward quench. (b) Work distributions $p_{Q}(W)$. (c) The joint distribution $\mathcal{P}(\beta, \Delta F)$ inferred from the estimates of $p_{Q}(W)$, together with the corresponding marginal distributions $\mathcal{P}(\beta)$ and $\mathcal{P}(\Delta F)$. (d) The fractional standard deviation $\sigma_{\beta} / \beta$ and bias $\left(\mu_{\beta}-\beta\right) / \beta$ of the $\beta$ estimates observed over 1000 simulated experiments, plotted against the actual $\beta$. The values of $T$ are shown in the figure. Unless stated otherwise, for all figures, the system parameters are $M=1000, U / J=0.1$, and $n=1$, the quench parameters are $\lambda_{i} \eta / J=0, \lambda_{f} \eta / J=0.5$, and $\tau J=1$, and the protocol parameters are $N_{\text {steps }}=500, N_{\text {meas }}=500$, and $T J=2.9 \pi$.

Here $\hat{b}_{k}^{\dagger}$ and $\hat{b}_{k}$ create and annihilate a phonon at quasimomentum $k, \omega_{k}=\sqrt{\epsilon_{k}\left(\epsilon_{k}+2 U n\right)}$ is the phonon dispersion written in terms of single-particle energies $\epsilon_{k}=2 J(1-\cos k a)$, and $\eta_{k}=\eta \sqrt{n \epsilon_{k} / M \omega_{k}} e^{-i k a c}$ is the relative coupling of each phonon mode to the impurity, with $a$ the lattice parameter.

With the Hamiltonians $\hat{H}_{S}$ and $\hat{V}$ in this form, the characteristic function $\chi_{Q}(u)$ can be calculated exactly (see Supplemental Material [33]) from time integrals of the form $\Lambda_{Q k}=\omega_{k} \int_{0}^{\tau} d t \lambda_{Q}(t) \exp \left(-i \omega_{k} t\right)$. The specific quench considered here is $\lambda_{Q}(t)=\lambda_{Q}(0)+\left[\lambda_{Q}(\tau)-\right.$ $\left.\lambda_{Q}(0)\right] \sin ^{2}(\pi t / 2 \tau)$.

We demonstrate the protocol first for a temperature corresponding to the typical energy scale of the system, $\beta J=1$, with the results shown in Fig. 1. In Fig. 1(a) we have plotted the known characteristic function values $\chi_{F}\left(u_{j}\right)$ for the forward quench. Also shown are the estimated values and associated errors from a single simulated experiment, consisting of $2 N_{\text {meas }}$ measurements at $N_{\text {steps }}$ times. In Fig. 1(b) we show the corresponding work distributions, both forward $p_{F}(W)$ and backward $p_{B}(W)$, obtained from exact and estimated values of $\chi_{F}\left(u_{j}\right)$ and $\chi_{B}\left(u_{j}\right)$, again with error bars. Figure 1(c) shows the joint distribution $\mathcal{P}(\beta, \Delta F)$ of $\beta$ and $\Delta F$ conditioned upon the estimates of $p_{Q}(W)$ obtained. From this, the marginal distribution $\mathcal{P}(\beta)$ for $\beta$, also shown, is calculated. The distribution of this example is consistent with the known value, containing uncertainty in $\beta$ of only a few percent. This can be reduced by increasing $N_{\text {steps }}$ or $N_{\text {meas }}$.
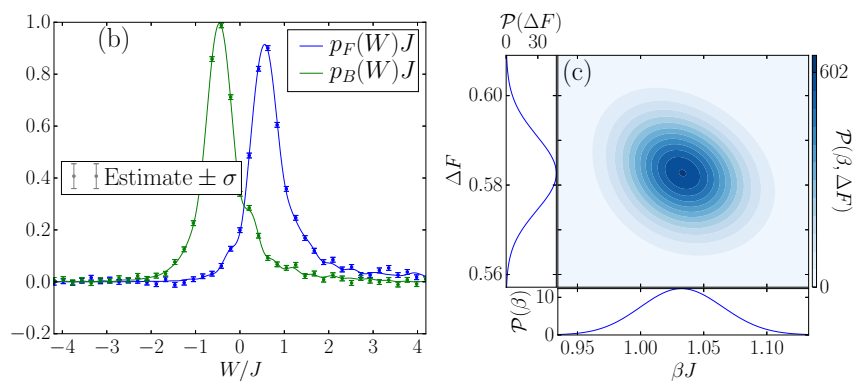

FIG. 2. Stronger interactions. The contents and parameters of this figure are identical to Figs. 1(b) and 1(c), but for a smaller $M=11$ system, featuring stronger interactions $U / J=4$ and $\lambda_{f} \eta / J=2$, and a shorter $\tau J=0.1$ quench and longer window-size $T J=15$.

The Bayesian prediction is remarkably well calibrated. For each of several values of $\beta$ between 1 and 50, we have simulated 1000 experiments of the above type. In Fig. 1(d) we plot the average mean and standard deviation of the inferred distribution $\mathcal{P}(\beta)$, with the average taken over the different experiments. This shows that consistent accuracy of a few percent can be obtained even as $\beta$ is reduced over two orders of magnitude. We also count the fraction of times in which the true $\beta$ value lies in each decile of the Bayesian prediction. Ideally this would be exactly 0.1 for each decile and our predictions conform to this, staying between 0.8 and 1.2. Plots of these values are given in the Supplemental Material [33].

\section{B. Stronger interactions}

We now study the success of the protocol when the bosons are more strongly interacting. In this case the Bogoliubov approach is not valid, and instead our analysis proceeds using time-evolving block decimation [37-39] to evolve a matrix product operator [40-42] representation of the state of the bosons. Using this we near exactly calculate the characteristic function $\chi_{Q}(u)$ for the exact Bose-Hubbard model [Eq. (4)] and interaction Hamiltonian. See the Supplemental Material [33] for details on this tensor network method [43].

The results for strongly interacting bosons, close to the critical point, are shown in Fig. 2. Compared to superfluid bosons, we see that, despite stronger impurity-boson coupling, the qubit dephasing takes place over a longer time scale due to the absence of a broad spectrum of low-energy excitations. Correspondingly the work distributions $p_{Q}(W)$, shown in Fig. 2(a), are more featured than for the superfluid case, with positive skewness resulting from a high-frequency shoulder. However, as shown in Fig. 2(b), the accuracy of the thermometry procedure remains largely indifferent to these changes.

\section{DISCUSSION}

We have shown that using a nonequilibrium probe overcomes two challenges in the thermometry of ultracold gases. First, the need to precisely control the internal energy of the probe on scales corresponding to the thermal energy of the system. Second, the need to understand the temperature dependence of the system's thermal state in advance. To demonstrate this, we showed that the temperature of bosons in 
a lattice could be estimated using the protocol, for temperatures in the nano-Kelvins or even lower. We also found that accuracy and precision are largely unaffected by moving close to a critical point, in this case the crossover between superfluid and insulating phases, a regime that is less well understood.

These advantages come at a cost, namely the need for exquisite control over the interaction between the probe qubit and the cold-atom system. Furthermore, it must be possible to perform a quench such that the nonequilibrium work fluctuations are comparable to the thermal energy of the system. We have argued that atomic impurity probes can be expected to satisfy the aforementioned requirements quite generally, and are therefore excellent candidates for generic thermometry of cold atoms at very low temperatures.

Instead of performing repeated measurements on a single qubit probe, multiple measurements could be performed simultaneously using multiple probes. These could be prepared in an array using a lattice and used either to reduce the number of measurements per probe or to probe a spatially varying temperature profile resulting from, e.g., heat currents during nonequilbrium transport $[44,45]$. Alternatively, if the qubit probe is implemented by interfering atoms passing through the system at shifted times, then a steady stream of atom probes could near continuously monitor the temperature of the system.
An essential assumption underlying our thermometry protocol is that the system is in thermal equilibrium. However, the protocol could potentially be used to assess whether this is the case. The protocol is used to find the most likely pairs $\beta$ and $\Delta F$ given that the Tasaki-Crooks relation holds, but it could also evaluate the likelihood that the relation is satisfied for any $\beta$ and $\Delta F$, thus allowing the testing of thermalization [46].

\section{ACKNOWLEDGMENTS}

The authors thank Sarah Al-Assam for her valuable assistance with the tensor network theory library [43]. T.H.J. and D.J. thank the National Research Foundation and the Ministry of Education of Singapore for support. M.T.M. acknowledges financial support from EPSRC via the Controlled Quantum Dynamics CDT. We gratefully acknowledge financial support from the Oxford Martin School Programme on Bio-Inspired Quantum Technologies, the European Research Council under the European Union's Seventh Framework Programme (FP7/2007-2013)/ERC Grant Agreement No. 319286 Q-MAC and the collaborative project QuProCS (Grant Agreement 641277), and the EPSRC through projects EP/K038311/1 and EP/J010529/1. Data produced by EPSRC funded work is contained in the source code corresponding to the arXiv submission of this article.
[1] T. H. Johnson, S. R. Clark, and D. Jaksch, EPJ Quantum Technol. 1, 1 (2014).

[2] L. A. Correa, M. Mehboudi, G. Adesso, and A. Sanpera, Phys. Rev. Lett. 114, 220405 (2015).

[3] A. E. Leanhardt, T. A. Pasquini, M. Saba, A. Schirotzek, Y. Shin, D. Kielpinski, D. E. Pritchard, and W. Ketterle, Science 301, 1513 (2003).

[4] I. Bloch, J. Dalibard, and W. Zwerger, Rev. Mod. Phys. 80, 885 (2008).

[5] J. F. Sherson, C. Weitenberg, M. Endres, M. Cheneau, I. Bloch, and S. Kuhr, Nature (London) 467, 68 (2010).

[6] J. Goold, T. Fogarty, N. Lo Gullo, M. Paternostro, and T. Busch, Phys. Rev. A 84, 063632 (2011).

[7] P. Haikka, J. Goold, S. McEndoo, F. Plastina, and S. Maniscalco, Phys. Rev. A 85, 060101 (2012).

[8] A. Sindona, J. Goold, N. Lo Gullo, S. Lorenzo, and F. Plastina, Phys. Rev. Lett. 111, 165303 (2013).

[9] M. Punk and S. Sachdev, Phys. Rev. A 87, 033618 (2013).

[10] A. Recati, P. O. Fedichev, W. Zwerger, J. von Delft, and P. Zoller, Phys. Rev. Lett. 94, 040404 (2005).

[11] T. H. Johnson, S. R. Clark, M. Bruderer, and D. Jaksch, Phys. Rev. A 84, 023617 (2011).

[12] P. Haikka, S. McEndoo, and S. Maniscalco, Phys. Rev. A 87, 012127 (2013).

[13] M. Bruderer and D. Jaksch, New J. Phys. 8, 87 (2006).

[14] C. Sabín, A. White, L. Hackermuller, and I. Fuentes, Sci. Rep. 4, 6436 (2014).

[15] D. Hangleiter, M. T. Mitchison, T. H. Johnson, M. Bruderer, M. B. Plenio, and D. Jaksch, Phys. Rev. A 91, 013611 (2015).

[16] P. O. Fedichev and U. R. Fischer, Phys. Rev. Lett. 91, 240407 (2003).
[17] H. Tasaki, arXiv:cond-mat/0009244.

[18] P. Talkner and P. Hanggi, J. Phys. A 40, F569 (2007).

[19] M. Campisi, P. Hänggi, and P. Talkner, Rev. Mod. Phys. 83, 771 (2011).

[20] J. Goold, M. Huber, A. Riera, L. del Rio, and P. Skrzypzyk, J. Phys. A: Math. Theor. 49, 143001 (2016).

[21] R. Dorner, S. R. Clark, L. Heaney, R. Fazio, J. Goold, and V. Vedral, Phys. Rev. Lett. 110, 230601 (2013).

[22] L. Mazzola, G. De Chiara, and M. Paternostro, Phys. Rev. Lett. 110, 230602 (2013).

[23] T. B. Batalhão, A. M. Souza, L. Mazzola, R. Auccaise, R. S. Sarthour, I. S. Oliveira, J. Goold, G. De Chiara, M. Paternostro, and R. M. Serra, Phys. Rev. Lett. 113, 140601 (2014).

[24] S. Palzer, C. Zipkes, C. Sias, and M. Köhl, Phys. Rev. Lett. 103, 150601 (2009).

[25] S. Will, T. Best, S. Braun, U. Schneider, and I. Bloch, Phys. Rev. Lett. 106, 115305 (2011).

[26] N. Spethmann, F. Kindermann, S. John, C. Weber, D. Meschede, and A. Widera, Phys. Rev. Lett. 109, 235301 (2012).

[27] J. Catani, G. Lamporesi, D. Naik, M. Gring, M. Inguscio, F. Minardi, A. Kantian, and T. Giamarchi, Phys. Rev. A 85, 023623 (2012).

[28] A. Sindona, J. Goold, N. L. Gullo, and F. Plastina, New J. Phys. 16, 045013 (2014).

[29] A. J. Roncaglia, F. Cerisola, and J. P. Paz, Phys. Rev. Lett. 113, 250601 (2014).

[30] G. De Chiara, A. J. Roncaglia, and J. P. Paz, New J. Phys. 17, 035004 (2015).

[31] G. Huber, F. Schmidt-Kaler, S. Deffner, and E. Lutz, Phys. Rev. Lett. 101, 070403 (2008). 
[32] S. An, J.-N. Zhang, M. Um, D. Lv, Y. Lu, J. Zhang, Z.-Q. Yin, H. Quan, and K. Kim, Nat. Phys. 11, 193 (2015).

[33] See Supplemental Material at http://link.aps.org/supplemental/ 10.1103/PhysRevA.93.053619 for a description of our Bayesian statistical approach, as well as details of our analytical and numerical calculations for the Bose-Hubbard model.

[34] J. F. Schaff, T. Langen, and J. Schmiedmayer, in Proceedings of the International School of Physics Enrico Fermi, edited by M. A. Kasevich and G. M. Tino (IOP Press, Amsterdam, 2014).

[35] D. Jaksch, C. Bruder, J. I. Cirac, C. W. Gardiner, and P. Zoller, Phys. Rev. Lett. 81, 3108 (1998).

[36] D. van Oosten, P. van der Straten, and H. T. C. Stoof, Phys. Rev. A 63, 053601 (2001).

[37] G. Vidal, Phys. Rev. Lett. 93, 040502 (2004).

[38] S. R. White and A. E. Feiguin, Phys. Rev. Lett. 93, 076401 (2004).
[39] T. H. Johnson, S. R. Clark, and D. Jaksch, Phys. Rev. E 82, 036702 (2010).

[40] F. Verstraete, J. J. García-Ripoll, and J. I. Cirac, Phys. Rev. Lett. 93, 207204 (2004).

[41] M. Zwolak and G. Vidal, Phys. Rev. Lett. 93, 207205 (2004).

[42] U. Schöllwock, Ann. Phys. 326, 96 (2011).

[43] S. Al-Assam, S. R. Clark, and D. Jaksch, Tensor network theory library, beta version 1.0.13, www.tensornetworktheory. org (2015).

[44] J.-P. Brantut, C. Grenier, J. Meineke, D. Stadler, S. Krinner, C. Kollath, T. Esslinger, and A. Georges, Science 342, 713 (2013).

[45] J. J. Mendoza-Arenas, T. Grujic, D. Jaksch, and S. R. Clark, Phys. Rev. B 87, 235130 (2013).

[46] J. Eisert, M. Friesdorf, and C. Gogolin, Nat. Phys. 11, 124 (2015). 